'Departamento de Salud Pública, Facultad de Medicina, CIEDE-UCSC, Universidad Católica de la Santísima Concepción. Concepción, Chile. 2Escuela de Kinesiología, Facultad de Salud, Universidad Santo Tomás, Chile

Pedagogía en Educación Física, Facultad de Educación, Universidad Autónoma de Chile. Chile.

"Instituto de Anatomía, Histología y Patología Facultad de Medicina, Universidad Austral de Chile. Valdivia, Chile.

Instituto de Farmacia, Facultad de Ciencias, Universidad Austral de Chile, Valdivia, Chile. ${ }^{6}$ Institute of Health and Wellbeing, University of Glasgow. Glasgow, United Kingdom. ${ }^{7}$ British Heart Foundation, Glasgow Cardiovascular Research Centre, University of Glasgow. Glasgow, United Kingdom ${ }^{8}$ Grupo de Investigación Calidad de Vida en distintas poblaciones, Departamento de Ciencias de la Educación, Universidad del Biobío, Chillán, Chile. 9Departamento de Nutrición y Dietética, Facultad de Farmacia, Universidad de Concepción. Concepción, Chile. Departamento de Psicología, Facultad de Ciencias Sociales, Universidad de Concepción. Concepción,

Chile.

'Departamento de Bioquímica Clínica e Inmunología. Facultad de Farmacia y Centro de Vida Saludable de la Universidad de Concepción. Concepción, Chile. ${ }^{12}$ Instituto de Nutrición y Tecnología de los Alimentos (INTA), Universidad de Chile. Chile. ${ }^{13}$ Centro de Investigación en Fisiología del EjercicioCIFE, Universidad Mayor. Santiago, Chile.

${ }^{14}$ Laboratorio de Rendimiento Humano, Grupo de Estudio en Educación, Actividad Física y Salud (GEEAFyS), Universidad Católica del Maule, Talca, Chile. ${ }^{15}$ Centro Vida Saludable, Universidad de Concepción. Concepción, Chile. ${ }^{16} \mathrm{Keiser}$ University, USA.

${ }^{7}$ Núcleo de Investigación Interdisciplinar en Salud (NIIS), Centro de Investigación en Gerontología Aplicada (CIGAP), Facultad de Salud, Universidad Santo Tomás, Chile. Nutricionista. bKinesiólogo. cProfesora de Biología y Química. 'Bioquímico. eProfesor de Educación Física. tPsicóloga. gLicenciado en Matemáticas. ${ }^{\mathrm{h} M s c}$ IPhD.

Trabajo no recibió financiamiento. Los autores declaran no tener conflictos de interés. Recibido el 2 de septiembre de 2019, aceptado el 23 de septiembre de 2020

Correspondencia a: Claudia Troncoso Pantoja Universidad Católica de la Santísima Concepción. Facultad de Medicina. Campus San Andrés. Alonso de Ribera 2850 Concepción, Chile. ctroncosop@ucsc.cl

\section{Prevalencia de fragilidad en personas mayores de Chile: resultados de la Encuesta Nacional de Salud 2016-2017}

\author{
CLAUDIA TRONCOSO-PANTOJA $\mathrm{A}^{1, \mathrm{a,h},}$ \\ YENY CONCHA-CISTERNAS ${ }^{2,3, \mathrm{~b}, \mathrm{~h}}$, \\ ANA MARÍA LEIVA-ORDOÑEZ $Z^{4, \mathrm{c}, \mathrm{h}}$, \\ MARÍA ADELA MARTIINEZ-SANGUINETTI ${ }^{5, \mathrm{d,h},}$ \\ FANNY PETERMANN-ROCHA ${ }^{6,7, a, h}$,

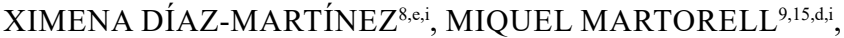 \\ GABRIELA NAZAR ${ }^{10,15, \mathrm{f}, \mathrm{i}}$, NATALIA ULLOA ${ }^{11, \mathrm{~d}, \mathrm{i}}$, \\ IGOR CIGARROA-CUEVAS ${ }^{2,17, b, i}$, CECILIA ALBALA ${ }^{12}$, \\ CARLOS MÁRQUEZ ${ }^{12, a, h}$, LYDIA LERA ${ }^{12,16, \mathrm{~g}}$, \\ CARLOS CELIS-MORALES $6,7,13,14, \mathrm{e}, \mathrm{i}$ \\ en representación del Grupo ELHOC \\ (Epidemiology of Lifestyle and Health Outcomes in Chile)
}

\section{Prevalence of frailty among Chilean older people}

Background: The assessment of frailty among older people could help to reduce its social and health burden. Aim: To determine and characterize the prevalence of frailty in Chilean older adults. Material and Methods: We studied 233 participants, aged $>60$ years, participating in the Chilean National Health Survey 2016-2017. Frailty was assessed using modified Fried criteria. Thus, people classified as frail should meet at least 3 out of the 5 criteria (low strength, low physical activity, low body mass index, slow walking pace and tiredness). Results: The prevalence of frailty was $10.9 \%$ (7.7\% for men and $14.1 \%$ for women). The prevalence of pre-frailty was $59.0 \%$ whereas $30.1 \%$ of participants were classified as robust. At the age of 80 years 58 and $62 \%$ of men and women were frail, respectively. These figures increased to 90 and $87 \%$ at the age of 90 years. The prevalence of pre-frailty increased from 43 to $92.1 \%$ among men and from $76 \%$ and $78 \%$ among women from the ages of 60 to 90 years, respectively. Conclusions: The prevalence of frailty increased markedly with age. It is important to implement prevention strategies to allow an early identification of high-risk individuals.

(Rev Med Chile 2020; 148: 1418-1426)

Key words: Aged; Frailty; Health Surveys; Prevalence. 


\section{L} a Organización Mundial de la Salud (OMS) ha proyectado un incremento de 900 millones de adultos mayores de 60 años para el año $2015^{1}$. En otras palabras, las poblaciones octogenarias y nonagenarias tendrán un importante incremento, lo que a su vez podría estar acompañado de un aumento de patologías asociadas a su condición fisiológica como patológica, originadas por restricciones en su movilidad, problemas físicos y mentales que caracterizan al síndrome de fragilidad ${ }^{2}$.

La fragilidad ha sido diagnosticada mediante el uso de diferentes criterios; uno de los más comunes es el fenotipo de Fried ${ }^{3}$, el que se basa en cinco componentes para definir esta condición (fuerza muscular, marcha, agotamiento, pérdida de peso y fatiga crónica), asociándose a un mayor riesgo de caídas, hospitalizaciones, discapacidad e incremento en el riesgo de mortalidad ${ }^{4}$.

La temprana identificación de esta condición, es esencial y necesaria para evitar situaciones adversas en aspectos relacionados al bienestar individual y económico de personas con síndrome de fragilidad y sus familias ${ }^{5}$. Se ha reportado que personas con fragilidad podrían retrasar su aparición si se sometieran a un test de screening que permita su temprana identificación e intervención ${ }^{6}$.

Se reconoce que alrededor de $40 \%$ de las personas mayores de 80 años presentan alguna limitación de capacidad funcional y que entre $6 \%$ a $11 \%$ es considerado frágil según la clasificación de Fried $^{7}$. En Latinoamérica y el Caribe, la prevalencia de fragilidad es de $19,6 \%$, con rangos que varían entre $7 \%$ a $42,6 \%{ }^{8}$. En Chile, datos aportados por el estudio realizado en la zona norte del país, reflejan un incremento con la edad, alcanzando $52,9 \%$ en las personas mayores entre 80 a 84 años ${ }^{9}$.

Considerando la necesidad e importancia de orientar las políticas y programas destinados a este grupo etario que presenta la identificación de fragilidad en Chile, el objetivo de esta investigación fue determinar y caracterizar la prevalencia de fragilidad en personas mayores de Chile.

\section{Materiales y métodos}

Diseño del estudio

El estudio utilizó datos de la Encuesta Nacional de Salud 2016-2017 (ENS 2016-2017). En términos generales, es una encuesta poblacional de corte transversal, probabilístico estratificado geográficamente, multietápico y de conglomerados, que permitió obtener la prevalencia de condiciones de salud en la población chilena mayor de 15 años, provenientes de zonas urbanas y rurales de 15 regiones del país. Esta investigación incluye una submuestra de la ENS 2016-2017, que incluyó a personas mayores (edad $\geq 60$ años) reclutadas en la Región Metropolitana $(n=295)$. De estas personas invitadas solo $255(87 \%)$ aceptaron participar. $\mathrm{Al}$ derivar la variable de fragilidad, 233 personas mayores contaban con información para todos los criterios de definición de fragilidad. Para ponderar los análisis y resultados obtenidos a población nacional la siguiente investigación aplicó los factores de expansión sugeridos por la ENS 20162017. Por lo tanto, luego de aplicar los factores de expansión, la muestra expandida incluida en este estudio representa de manera teórica a 1.053.597 personas mayores en Chile.

El protocolo de estudio fue aprobado por el Comité de Ética de la Facultad de Medicina de la Pontificia Universidad Católica de Chile y todos los participantes dieron su consentimiento informado previo a la recolección de datos ${ }^{10}$.

\section{Clasificación de la población según fragilidad}

La fragilidad se evaluó en base a la escala de fenotipo de Fried modificado, que incluye fuerza muscular de prensión $(<20$ percentil), inactividad actividad física $(<150 \mathrm{~min} / \mathrm{semana}$, autoreportado), ritmo de caminata lento (autoreportado), índice de masa corporal (IMC) $\left(<23,0 \mathrm{~kg} / \mathrm{m}^{2}\right)$ e incapacidad de realizar tareas de la vida cotidiana (autoreportado). Para esta investigación y siguiendo una interpretación clásica de fragilidad, personas mayores con tres o más de las cinco condiciones fueron clasificadas como frágiles, aquellas con dos o menos de cinco criterios fueron clasificadas como pre-frágil y personas sin ninguno de estos criterios fueron clasificados como robustos ${ }^{11,13}$.

\section{Mediciones y recolección de datos}

Las variables de tipo sociodemográficas fueron recolectadas mediante auto-reporte de cada participante. Estas incluyeron edad, sexo, lugar de residencia (urbano/rural), nivel educacional (básica, media y técnica/universitaria) y diagnóstico de enfermedades. La existencia de multimorbilidad fue determinada en base al reporte 
de 15 enfermedades medicamente diagnosticadas (diabetes mellitus tipo 2, hipertensión arterial, colesterol elevado, síndrome metabólico, infarto agudo al miocardio, accidente cerebrovascular, enfermedad cardiovascular periférica, enfermedad cardiovascular, cáncer, osteoporosis, insuficiencia renal, enfermedad pulmonar obstructiva crónica, asma, apnea del sueño y depresión) las cual fueron categorizadas en 4 grupos (sin presencia de enfermedades, 1 enfermedad, 2 enfermedades $y \geq 3$ enfermedades). La percepción de salud y bienestar fue recolectada mediante cuestionario.

El peso corporal $(\mathrm{kg})$ y talla $(\mathrm{m})$ fueron medidos mediante protocolos estandarizados y utilizados para derivar el IMC $\left(\mathrm{kg} / \mathrm{m}^{2}\right)$. El punto de corte para el IMC consideró el grupo etario, clasificando como bajo peso al valor $\leq 22,9 \mathrm{~kg} / \mathrm{m}^{2}$; normal: $23,0-27,9 \mathrm{~kg} / \mathrm{m}^{2}$; sobrepeso: $28,0-31,9 \mathrm{~kg} /$ $\mathrm{m}^{2} \mathrm{y}$ obesidad $\geq 32,0 \mathrm{~kg} / \mathrm{m}^{2}{ }^{14}$. La obesidad central fue definida utilizando el perímetro de cintura (PC), utilizando los puntos de corte $\geq 102 \mathrm{~cm}$ para varones $\mathrm{y} \geq 88 \mathrm{~cm}$ para mujeres ${ }^{15}$.

Los niveles de actividad física, el tiempo destinado a las actividades de transporte y las actividades de intensidad moderada o vigorosa de la población, fueron determinados con el cuestionario "Global Physical Activity Questionnaire" ${ }^{16}$. Se consideró como punto de corte para inactividad física un gasto energético menor a $600 \mathrm{METs} / \mathrm{minutos} / \mathrm{semana}$. Los niveles de sedentarismo fueron determinados mediante el auto-reporte de tiempo destinado a actividades que involucren estar sentado o reclinado durante el tiempo libre o de trabajo mediante el mismo cuestionario. Fuerza de prensión manual fue medida mediante un test de dinamometría de prensión realizado en ambas manos y el valor máximo registrado fue utilizado para determinar prensión máxima.

\section{Análisis estadísticos}

Los datos de caracterización de población y componentes de fragilidad estudiados son presentados como promedios y porcentajes con sus respectivos intervalos de confianza de $95 \%$. Todos los análisis fueron ajustados por el factor de expansión. La muestra expandida corresponde a la extrapolación a población nacional realizada con los factores de expansión sugeridos por la ENS 2016-2017. Se utilizo el software STATA MP versión 15 para realizar los análisis estadísticos.

\section{Resultados}

La Tabla 1 presenta las características generales de la población estudiada según nivel de fragilidad (robusto, pre-frágil y frágil). El perfil de las personas mayores identificadas con fragilidad se caracterizó por una mayor prevalencia en mujeres, donde $64,9 \%$ de ellas presentó fragilidad en comparación a $35,1 \%$ de los hombres. Con relación a nivel educacional, personas con niveles más bajos de educación formal ( $<8$ años) presentaron la prevalencia más alta de fragilidad $(63,1 \%)$ mientras que solo $9,6 \%$ de las personas mayores con niveles altos de educación presentó fragilidad. Para estado nutricional la prevalencia de fragilidad fue marcadamente más alta en personas con bajo peso o enflaquecido $(22,1 \%)$ en comparación a personas mayores clasificadas como robustas donde no se presentaron casos de bajo peso. También cabe destacar que de las personas mayores clasificadas como robustas, $80,7 \%$ exhibieron sobrepeso y obesidad en comparación a $45 \%$ de las personas clasificadas como frágiles. Sin embargo, estas diferencias no se observaron para obesidad central. Otra marcada diferencias entre personas robustas y frágiles fue observada para el auto-reporte de bienestar, en que $83,5 \%$ de las personas clasificadas como robustas refirieron presentar un buen estado de salud y bienestar en comparación a solo $27,5 \%$ de las personas clasificadas como frágiles, es más, $24,7 \%$ de las personas frágiles reportaron un mal estado de salud o bienestar. En términos de multimorbilidad se observó que $64 \%$ de las personas mayores frágiles presenta tres o más enfermedades en comparación a $41 \%$ de la población clasificada como robusta. Estos resultados concuerdan con la prevalencia de hipertensión, enfermedades cardiovasculares y cáncer, que fue mayor en personas con fragilidad versus aquellas robustas, aunque la prevalencia de diabetes fue mas baja en personas mayores con fragilidad. También se destaca que, en comparación a las personas robustas, los que presentan fragilidad exhibieron mayor edad y son más sedentarios, además de presentar un menor peso corporal, IMC, perímetro de cintura, fuerza muscular y niveles de actividad física (Tabla 1).

La prevalencia de subcomponentes de fragilidad total según sexo se presenta en la Tabla 2. De los cinco subcomponentes para la definición de 
Tabla 1. Características de la población según fragilidad

\begin{tabular}{|c|c|c|c|}
\hline & Robusto & Pre-Frágil & Frágil \\
\hline Muestra encuestada, $(n)^{*}$ & 67 & 139 & 27 \\
\hline Muestra expandida $(n)^{*}$ & 317,756 & 621,235 & 114,605 \\
\hline Edad, (años) & $66,5(64,4 ; 68,5)$ & $69,7(67,5 ; 72,0)$ & $76,5(72,4 ; 80,6)$ \\
\hline $\begin{array}{l}\text { Grupo etario } \\
60-65 \text { años } \\
66 \text { a } 70 \text { años } \\
71 \text { a } 75 \text { años } \\
76 \text { a } 80 \text { años } \\
>80 \text { años }\end{array}$ & $\begin{array}{c}59.7(43,1 ; 74,3) \\
12,7(5,7 ; 26,0) \\
18,2(9,6 ; 31,6) \\
4,8(1,4 ; 14,9) \\
4,7(1,4 ; 14,6)\end{array}$ & $\begin{array}{c}39,4(28,5 ; 51,6) \\
22,6(15,2 ; 32,2) \\
12,7(7,5 ; 20,5) \\
10,6(6,0 ; 18,1) \\
14,7(7,4 ; 27,1)\end{array}$ & $\begin{array}{c}16,2(4,6 ; 43,8) \\
6,1(1,6 ; 21,1) \\
20,5(6,5 ; 48,7) \\
18,9(6,3 ; 44,8) \\
38,3(19,0 ; 62,2)\end{array}$ \\
\hline $\begin{array}{l}\text { Sexo }(\%) \\
\text { Hombres } \\
\text { Mujeres }\end{array}$ & $\begin{array}{l}61,1(44,5 ; 75,6) \\
38,9(24,4 ; 55,5)\end{array}$ & $\begin{array}{l}46,8(35,4 ; 58,4) \\
53,2(41,5 ; 64,5)\end{array}$ & $\begin{array}{l}35,1(16,1 ; 60,5) \\
64,9(39,4 ; 83,9)\end{array}$ \\
\hline $\begin{array}{l}\text { Nivel educacional (\%) } \\
<8 \text { años } \\
8-12 \text { años } \\
>12 \text { años }\end{array}$ & $\begin{array}{l}24,4(14,1 ; 38,7) \\
52,8(35,5 ; 69,4) \\
22,8(10,1 ; 43,8)\end{array}$ & $\begin{array}{l}37,2(27,1 ; 48,5) \\
35,9(25,7 ; 47,5) \\
26,9(17,4 ; 39,2)\end{array}$ & $\begin{array}{c}63,1(37,5 ; 83,0) \\
27,3(11,0 ; 53,1) \\
9,6(1,5 ; 42,1)\end{array}$ \\
\hline $\begin{array}{l}\text { Características antropométricas } \\
\text { Talla (metros) } \\
\text { Peso }(\mathrm{Kg}) \\
\text { IMC }\left(\mathrm{kg} / \mathrm{m}^{2}\right) \\
\text { Perímetro de cintura, }(\mathrm{cm}) \\
\text { Fuerza de prensión }(\mathrm{kg}) \\
\text { Fuerza de prensión }(\mathrm{kg} / \text { peso) }\end{array}$ & $\begin{array}{c}1,63(1,59 ; 1,66) \\
78,3(74,3 ; 82,2) \\
29,5(28,45 ; 30,7) \\
99,4(96,8 ; 102,1) \\
36,4(30,9 ; 41,9) \\
0,46(0,40 ; 0,52)\end{array}$ & $\begin{array}{l}1,59(1,58 ; 1,61) \\
73,4(70,9 ; 75,8) \\
28,9(27,9 ; 29,8) \\
97,1(94,2 ; 100,0) \\
25,0(21,9 ; 28,1) \\
0,34(0,30 ; 0,38)\end{array}$ & $\begin{array}{c}1,54(1,50 ; 1,58) \\
63,9(56,8 ; 70,9) \\
27,0(23,9 ; 30,1) \\
94,8(87,8 ; 101,8) \\
16,0(12,3 ; 19,6) \\
0,26(0,19 ; 0,32)\end{array}$ \\
\hline $\begin{array}{l}\text { Estado nutricional (\%) } \\
\text { Bajo peso } \\
\text { Normal } \\
\text { Sobrepeso } \\
\text { Obeso }\end{array}$ & $\begin{array}{c}0 \\
19,3(10,6 ; 32,4) \\
43,0(26,9 ; 60,6) \\
37,7(22,0 ; 56,5)\end{array}$ & $\begin{array}{c}6,3(2,7 ; 13,6) \\
20,3(12,6 ; 31,1) \\
53,2(41,7 ; 64,4) \\
20,2(12,4 ; 31,2)\end{array}$ & $\begin{array}{c}22,1(8,4 ; 46,7) \\
32,9(15,3 ; 57,2) \\
27,4(10,7 ; 54,2) \\
17,6(5,5 ; 43,9)\end{array}$ \\
\hline $\begin{array}{l}\text { Obesidad central, (\%) } \\
\text { Normal } \\
\text { Obeso central }\end{array}$ & $\begin{array}{l}42,8(26,5 ; 60,8) \\
57,2(39,2 ; 73,5)\end{array}$ & $\begin{array}{l}43,6(32,5 ; 55,4) \\
56,4(44,6 ; 67,5)\end{array}$ & $\begin{array}{l}46,7(25,0 ; 69,8) \\
53.3(30,2 ; 75,0)\end{array}$ \\
\hline $\begin{array}{l}\text { Autobienestar en salud (\%) } \\
\text { Mal } \\
\text { Regular } \\
\text { Bien }\end{array}$ & $\begin{array}{c}0 \\
16,5(8,3 ; 29,9) \\
83,5(70,1 ; 91,7)\end{array}$ & $\begin{aligned} 5,2 & (2,2 ; 11,8) \\
34,5 & (23,7 ; 47,2) \\
60,3 & (48,1 ; 71,4)\end{aligned}$ & $\begin{array}{c}24,7(9,5 ; 50,7) \\
47,9(25,8 ; 70,8) \\
27,5(11,4 ; 52,8)\end{array}$ \\
\hline $\begin{array}{l}\text { Enfermedades (\%) } \\
\text { Hipertensión } \\
\text { Diabetes } \\
\text { ECV } \\
\text { Cáncer } \\
\text { EPOC }\end{array}$ & $\begin{array}{c}57,5(39,1 ; 74,0) \\
26,4(13,8 ; 44,5) \\
24,0(12,2 ; 41,8) \\
0,6(0,1 ; 3,0) \\
0\end{array}$ & $\begin{array}{l}67,5(56,5 ; 76,8) \\
30,5(20,9 ; 42,1) \\
25,6(17,4 ; 36,0) \\
8,6(4,3 ; 16,4) \\
5,9(2,3 ; 14,0)\end{array}$ & $\begin{array}{c}74,5(47,2 ; 90,5) \\
19,2(6,1 ; 46,5) \\
41,8(21,3 ; 65,6) \\
32,6(14,4 ; 58,2) \\
3,6(0,4 ; 22,4)\end{array}$ \\
\hline $\begin{array}{l}\text { Multimorbilidad (\%) } \\
\text { Sin presencia de patología } \\
1 \text { patología } \\
2 \text { patologías } \\
\geq 3 \text { patologías }\end{array}$ & $\begin{array}{c}17,0(6,2 ; 39,1) \\
21,5(11,6 ; 36,3) \\
20,5(9,1 ; 39,9) \\
41,0(25,7 ; 58,3)\end{array}$ & $\begin{array}{c}10,0(5,1 ; 18,5) \\
19,1(11,3 ; 30,4) \\
17,6(9,5 ; 30,2) \\
53,3(41,7 ; 64,6)\end{array}$ & $\begin{array}{c}0 \\
6,2(1,1 ; 29,2) \\
29,8(12,8 ; 55,2) \\
64,0(39,4 ; 82,9)\end{array}$ \\
\hline $\begin{array}{l}\text { Actividad física (AF) } \\
\text { Inactividad física (\%) } \\
\text { AF total (MET/min/día) } \\
\text { Tiempo sedente (min/día) }\end{array}$ & $\begin{array}{c}0 \\
1.550,9(1025,5 ; 2076,2) \\
132,6(99,5 ; 165,7)\end{array}$ & $\begin{array}{c}30,5(21,2 ; 41,7) \\
565,2(345,3 ; 785,0) \\
199,0(164,5 ; 234,4)\end{array}$ & $\begin{array}{c}62,1(37,0 ; 82,1) \\
87,0(34,2 ; 139,9) \\
308,8(25.8 ; 391,8)\end{array}$ \\
\hline
\end{tabular}

Datos presentados como promedio o prevalencia expandidas a población nacional y sus respectivos $95 \%$ de intervalos de confianza. *Muestra encuestada corresponde a la población encuestada por la ENS y que fue incluida en este estudio. La muestra expandida corresponde a la extrapolación a población nacional realizada con los factores de expansión sugeridos por la ENS 2016-2017. EPOC: Enfermedad Pulmonar Obstructiva Cronica; ECV: Enfermedades Cardiovasculares; Diabetes: Diabetes Mellitus tipo 2: AF: Actividad Física. 
fragilidad, los que presentaron una mayor prevalencia fueron bajos niveles de actividad física $(44 \%)$, seguidos por un ritmo de marcha lento $(41,2 \%)$ y debilidad muscular $(21,9 \%)$; mientras que el criterio que presento la prevalencia más baja fue agotamiento $(5,4 \%)$. Estas prevalencias por subcomponentes de fragilidad fueron mayores en mujeres que en hombres, a excepción de debilidad muscular cuya prevalencia fue mayor en hombres (Tabla 2).

La Figura 1 presenta la prevalencia según fenotipos de fragilidad según sexo. El 37,0\% versus 23,4 de hombres y mujeres respectivamente fueron clasificados como robustos. Las mujeres presentaron una prevalencia de pre-fragilidad levemente más alta que los hombres $(62,6 \%$ versus $55,4 \%$, respectivamente). Mientras que la prevalencia de fragilidad en mujeres $(14,1 \%)$ fue el doble de la observada para hombres $(7,7 \%)$.

$\mathrm{Al}$ analizar la prevalencia de fragilidad por edad (Figura 2), se observó un drástico incremento en la prevalencia, en hombres y mujeres. La prevalencia de fragilidad fue de $9,7 \%$ y $4,4 \%$ en hombres y mujeres a los 60 años, respectivamente, alcanzando una prevalencia de $98,3 \%$ y $96,1 \%$ en mujeres y hombres a los 100 años. En cuanto a la prevalencia de pre-fragilidad (Figura 3), esta se incrementa con los años de manera más acelerada en hombres, aumentando de $42,1 \%$ a $97,8 \%$ entre los 60 y 100 años, respectivamente. Mientras que para mujeres aumentó de $70,4 \%$ a $80,0 \%$ entre los 60 y 100 años, de manera respectiva.

Tabla 2. Prevalencia de subcomponentes de fragilidad por sexo

\begin{tabular}{|lccc|}
\hline Subcomponentes de fragilidad (\%) & Total & Hombres & Mujeres \\
\hline Debilidad muscular & $21,9(15,4 ; 30,3)$ & $24,7(14,3 ; 39,0)$ & $19,3(12,1 ; 29,3)$ \\
Baja actividad física & $44,0(39,9 ; 48,2)$ & $37,5(31,3 ; 44,2)$ & $49,9(44,9 ; 55,1)$ \\
\hline IMC $\left(\leq 23,0 \mathrm{~kg} / \mathrm{m}^{2}\right)$ & $9,3(7,4 ; 11,8)$ & $9,1(6,3 ; 13,0)$ & $9,6(7,0 ; 12,9)$ \\
Agotamiento & $5,4(4,5 ; 6,5)$ & $4,1(2,9 ; 5,8)$ & $6,7(5,3 ; 8,3)$ \\
\hline Ritmo de marcha lento & $41,2(37,2 ; 45,3)$ & $34,9(28,9 ; 41,3)$ & $47,1(42,0 ; 52,2)$ \\
\hline
\end{tabular}

Datos presentados como prevalencia expandidas a población nacional y sus respectivos $95 \%$ IC.

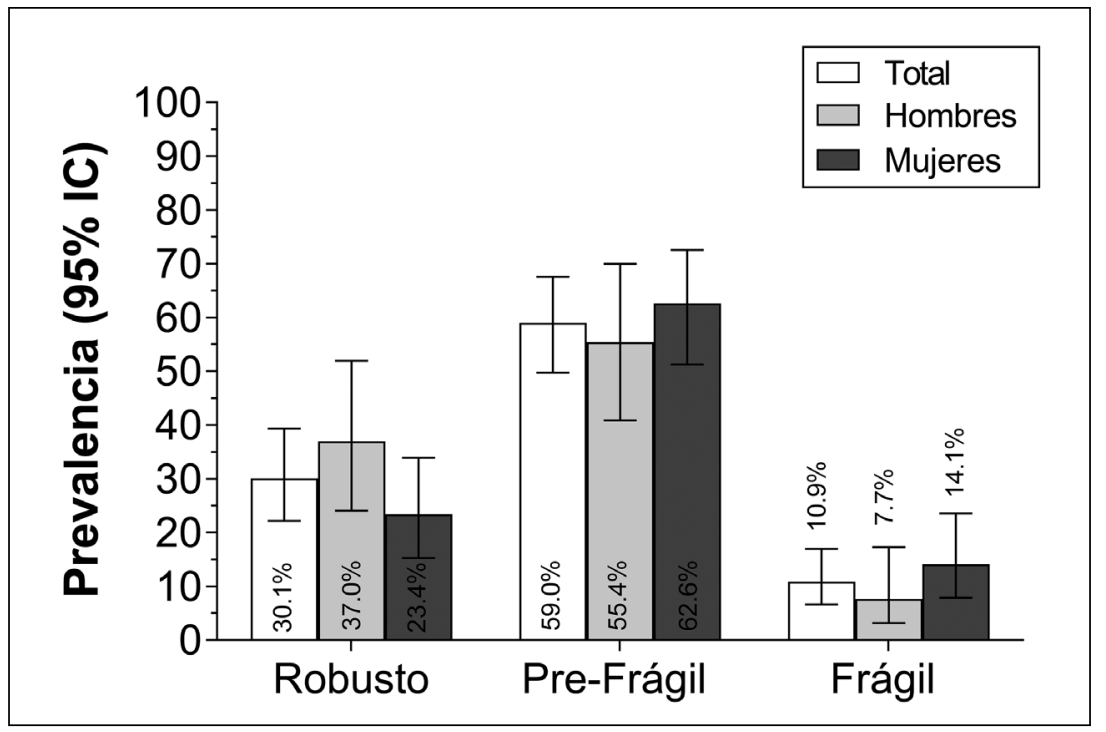

Figura 1. Prevalencia de fragilidad según sexo en adultos mayores en Chile. Datos presentados como prevalencia expandida a población nacional y sus $95 \%$ de intervalos de confianza según sexo. 

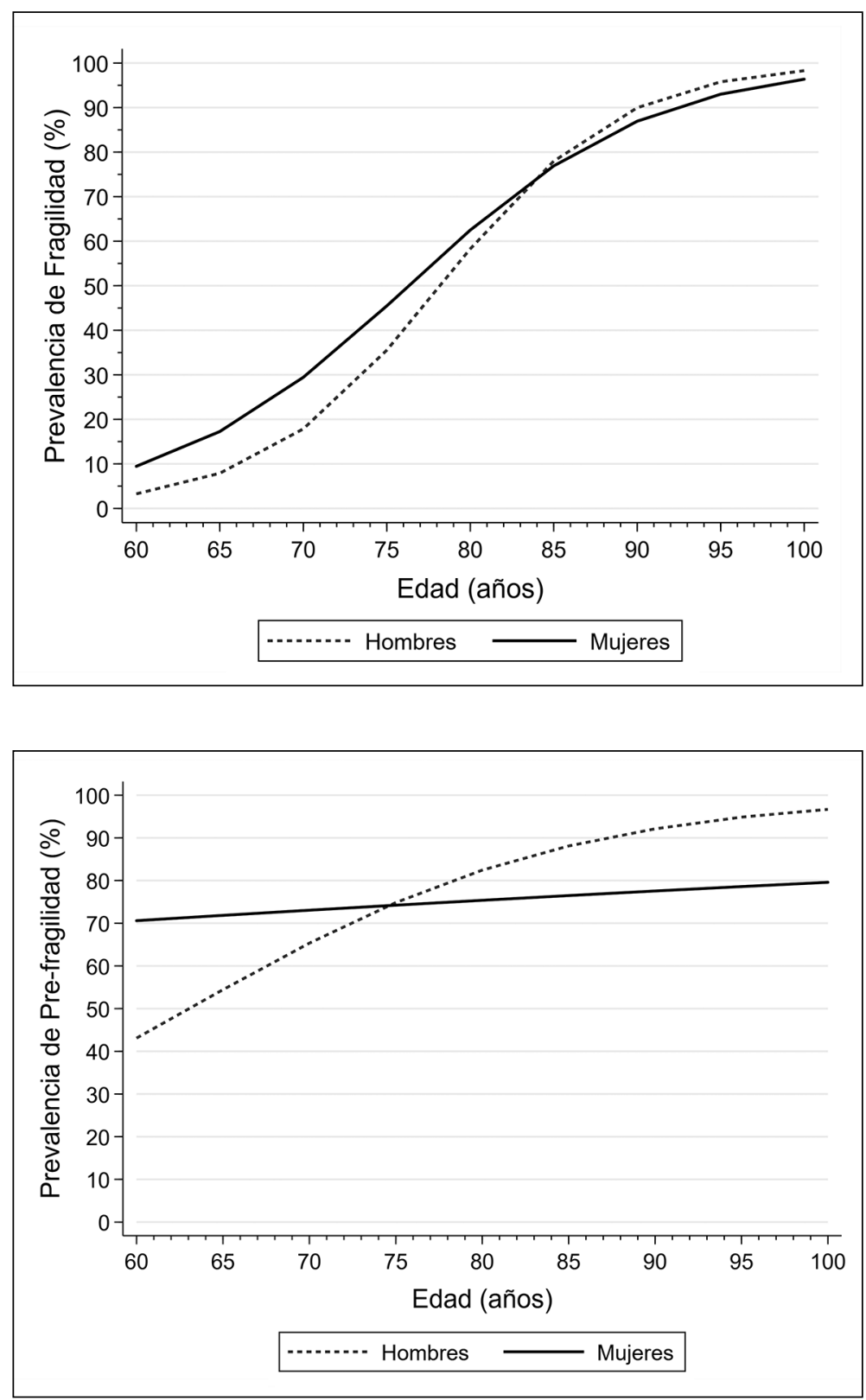

Figura 2. Prevalencia de fragilidad en población adulta mayor en Chile según edad. Datos presentados como prevalencia expandida a población nacional para mujeres (línea negra continua) y hombres (línea punteada) según edad en años.
Figura 3. Prevalencia de Pre-fragilidad en población adulta mayor en Chile según edad. Datos presentados como prevalencia expandida a población nacional para mujeres (línea negra continua) y hombres (línea punteada) según edad en años.

\section{Discusión}

Siguiendo la metodología de Fried, nuestro estudio identificó que $10,9 \%$ de la población chilena mayor de 60 años presentó fragilidad $(\geq 3$ criterios) y $59,0 \%$ pre-fragilidad (1-2 criterios). $\mathrm{Al}$ comparar nuestros resultados con la realidad local, los valores obtenidos para fragilidad son concordantes con los identificados en el estudio prospectivo ALEXANDROS, realizado por Albala y cols. En dicho estudio, participaron personas mayores de 60 años de Santiago, Chile, en un seguimiento que se extendió hasta 15 años y que utilizó como referencia diagnóstica el fenotipo de fragilidad, comprendido como dinamometría de agarre débil, la pérdida de peso involuntaria, fatiga 
o agotamiento, el sentarse en silla por cinco veces o velocidad lenta para caminar y la dificultad para caminar (baja actividad física). La investigación presenta como resultados una prevalencia de $13,9 \%$ de fragilidad ${ }^{17}$. Sin embargo, a excepción de la diferencia de géneros, nuestra prevalencia de fragilidad no es coincidente a otros estudios nacionales ${ }^{9,18}$. La investigación liderada por Tapia y cols., desarrollada en Antofagasta y que empleó para el diagnóstico de fragilidad la pérdida de peso involuntario superior a $4,5 \mathrm{~kg}$. previo al último año, la disminución en $20 \%$ de la fuerza de agarre de la mano dominante, medido con un dinamómetro ajustado según sexo e IMC; la limitada resistencia (autopercepción de fatiga fácil frente a las actividades habituales) y la actividad física (autoreporte), presentó una población con fragilidad que correspondió a 4,5\% de los sujetos estudiados ${ }^{9}$. También se mantiene una diferencia en la prevalencia de fragilidad $(24,6 \%)$ encontrada por Palomo y cols. en estudio de corte transversal aplicado a personas mayores de 65 años de la región del Maule y que utilizaron los criterios de Fried para la identificación de fragilidad ${ }^{18}$. Esta desigualdad de resultados podría presentar una respuesta en las diferencias propias de la población estudiada o las referencias utilizadas para el diagnóstico de fragilidad. A nivel global, nuestros resultados son cercanos a los identificados por Gleize y cols., en un estudio de prevalencia de tipo prospectivo en población adulta mayor, atendida de manera ambulatoria en Francia (10,9\% para usuarios frágiles $)^{19}, 12,1 \%$ identificado por Espinoza y cols. en estudio de cohorte desarrollado en mexicanos radicados en Norteamérica ${ }^{20} \mathrm{o}$ la prevalencia de $10,3 \%$ reconocida en población urbana española ${ }^{21}$, estudios que fueron realizados con estrategias metodológicas para la identificación de fragilidad similares a las presentadas en nuestro artículo.

De manera tradicional, se reconoce una marcada diferencia por sexos en la presentación de la fragilidad, acentuando una mayor prevalencia en las mujeres ${ }^{22,23}$. Nuestros resultados son consistentes a esta tendencia, al casi duplicar la presencia de fragilidad en mujeres en comparación con los hombres ( $14,1 \%$ y $7,7 \%$, respectivamente), valores que se acercan a $16,4 \%$ en mujeres y $8,7 \%$ en hombres encontrados por el estudio ALEXANDROS en Chile ${ }^{17}$ y también reportado en la revisión liderada por Shamliyan y cols. ${ }^{24}$. La mayor prevalencia en- contrada en mujeres se fundamentaría en las manifestaciones especialmente físicas del síndrome de fragilidad, asociadas a menor condición física, mayor comorbilidad, además de una reducción en la movilidad y la fuerza motriz originado por una menor masa muscular y una mayor infiltración grasa en su composición muscular ${ }^{25}$.

En cuanto a la pre-fragilidad, $59,0 \%$ de nuestra población se identifica con esta condición, situación que es similar a 54,3\% encontrado en el estudio dirigido Sousa-Santos y cols. en población de Portugal ${ }^{26}$, pero que se distancia a 40,9\% encontrado en estudio realizado en Perú ${ }^{27}$ o 28,1\% encontrado por Op het Veld y cols. en Países Bajos $^{28}$. Estas diferencias pueden estar dadas por la disimilitud entre los criterios utilizados para determinar fragilidad entre estudios.

Si bien este estudio presenta fortalezas, como lo es la utilización de métodos estandarizados para la determinación de fragilidad, este no está ajeno a limitaciones. Uno de ellos, es la falta de representatividad a nivel nacional ya que la muestra incluida en este estudio de fragilidad corresponde a personas mayores residentes de la Región Metropolitana, cuyas características podrían ser diferentes a aquellas personas mayores pertenecientes a otras regiones del país. Si bien se aplicó un factor de expansión para extender esta muestra a población nacional, esta es solo una estimación teórica, que podría sub o sobre valorar la verdadera prevalencia de fragilidad en personas mayores de otras regiones del país. Otro aspecto que considerar, en la interpretación de estos resultados, es la modificación de los criterios del fenotipo de Fried. Debido a la ausencia de ciertas mediciones se utilizaron variables alternativas y auto reportadas, lo cual limita la comparación con otros estudios realizados a nivel nacional e internacional.

\section{Conclusiones}

Nuestro estudio entrega evidencia que un gran porcentaje de personas mayores en Chile son frágiles y que esta prevalencia aumenta marcadamente en la medida que avanza la edad. Considerando que Chile liderara el incremento de la población mayor en comparación a otros países latinoamericanos, es imprescindible implementar medidas preventivas que permitan retrasar la aparición del 
síndrome de fragilidad, ya que esto se puede reducir la morbimortalidad en personas de este grupo etario, además de minimizar su dependencia y el riesgo de hospitalización, mejorando de esta manera su calidad de vida.

Agradecimientos: Se agradece a los participantes de la ENS 2016-2017 como también así al MINSAL y la Pontificia Universidad Católica de Chile que lideraron la ENS 2016-2017.

\section{Referencias}

1. Organización Mundial de la Salud. Envejecimiento y salud. Datos y cifras. 2018. Disponible en https://www. who.int/es/news-room/fact-sheets/detail/envejecimiento-y-salud [Consultado en abril de 2020].

2. Organización Mundial de la Salud. Envejecimiento y ciclo de vida. Disponible en https://www.who.int/ageing/ about/facts/es / [Consultado en abril de 2020].

3. Iriarte E, Araya AX. Criterios de fragilidad en personas mayores que viven en la comunidad: una actualización de la literatura. Rev Med Chile 2016; 144(11): 1440-7.

4. Carrasco M. Fragilidad: Un síndrome geriátrico en evolución. Disponible en: https://medicina.uc.cl/publicacion/fragilidad-sindrome-geriatrico-evolucion/ [Consultado en abril de 2020].

5. Romero AJ. Fragilidad: un síndrome geriátrico emergente. Medisur 2010; 8 (6): 81-90.

6. Tello-Rodríguez T, Varela-Pinedo L. Frailty in older adults: detection, community-based intervention, and decision-making in the management of chronic illnesses. Rev Perú Med Exp Salud Publica 2016; 33(2): 328-34.

7. García C. Evaluación y cuidado del adulto mayor frágil. Rev Med Clin Condes 2012; 23(1): 36-41.

8. Da Mata FA, Pereira PP, Andrade KR, Figueiredo AC, Silva MT, Pereira MG. Prevalence of Frailty in Latin America and the Caribbean: A Systematic Review and Meta-Analysis. PLoS One 2016; 11(8): e0160019.

9. Tapia C, Valdivia-Rojas Y, Varela H, Carmona A, Iturra $\mathrm{V}$, Jorquera M. Indicadores de fragilidad en adultos mayores del sistema público de salud de la ciudad de Antofagasta. Rev Med Chile 2015; 143 (4): 459-66.

10. Minsal. Orientación Técnica de Atención Integral para personas mayores frágiles en Unidades Gerátricas de Agudos (UGA). 2018. Disponible en https://www. minsal.cl/wp-content/uploads/2019/01/Orientaci\%C3\%B3n-T\%C3\%A9cnica-de-Atenci\%C3\%B3n-Integral-para-Personas-Mayores-Fr\%C3\%Algiles-en-Uni-
dades-Geri\%C3\%A1tricas-de-AgudosUGA.pdf [Consultado en abril de 2020].

11. Romero AJ. Fragilidad: un síndrome geriátrico emergente. Medisur 2010; 8 (6): 81-90.

12. Ministerio de Salud. Departamento de Epidemiología. Disponible en http://epi.minsal.cl/resultados-encuestas/ [Consultado en abril de 2020].

13. Mancilla E, Ramps S, Morales P. Fuerza de prensión manual según edad, género y condición funcional en adultos mayores chilenos entre 60 y 91 años. Rev Med Chile 2016; 144 (5): 598-603.

14. Yataco-Vicente JA, Araujo-Castillo RV. Necesidad de una aproximación integral al estado nutricional del adulto mayor. Rev Cubana Salud Pública 2018; 44 (3): e1248.

15. Moreno M. Definición y clasificación de la obesidad. Rev Med Clin Condes 2012; 23 (2): 124-8.

16. Armstrong T, Bull F. Development of the World Health Organization Global Physical Activity Questionnaire (GPAQ). J Public Health 2006;14 (2): 66-70.

17. Albala C, Lera L, Sánchez H, Angel B, Márquez C, Arroyo P, et al. Frequency of frailty and its association with cognitive status and survival in older Chileans. Clin Interv Aging 2017; 12:995-1001.

18. Palomo I, Giacaman RA, León S, Lobos G, Bustamante M, Wehinger S, Tapia JC et al. Analysis of the characteristics and components for the frailty syndrome in older adults from central Chile. The PIEI-ES study. Arch Gerontol Geriatr 2019; 80: 70-5.

19. Gleize F, Zmudka J, Lefresne Y, Serot JM, Berteaux B, Jouanny P. Fragility assessment in primary care: which tools for predicting what? Geriatr Psychol Neuropsychiatr Vieil 2015; 13(3): 289-97.

20. Espinoza SE, Jung I, Hazuda H. Frailty transitions in the San Antonio Longitudinal Study of Aging. J Am Geriatr Soc 2012; 60 (4): 652-60.

21. Castell MV, Otero A, Sánchez MT, Garrido A, González JI, Zunzunegui V. Prevalencia de fragilidad en una población urbana de mayores de 65 años y su relación con comorbilidad y discapacidad. Atención Primaria 2010; 42 (10): 520-7.

22. Ntanasi E, Yannakoulia M, Mourtzi N, Vlachos GS, Kosmidis $\mathrm{MH}$, Anastasiou CA et al. Prevalence and Risk Factors of Frailty in a Community-Dwelling Population: The HELIAD Study. J Aging Health 2020; 32 (1): 14-24.

23. Lourenço RA, Moreira VG, Banhato EFC, Guedes DV, Silva KCAD, Delgado FEDF. et al. Prevalence of frailty and associated factors in a community-dwelling older people cohort living in Juiz de Fora, Minas Gerais, Brazil: Fibra-JF Study. Cien Saude Colet 2019; 24 (1): 35-44. 
24. Shamliyan T, Talley KM, Ramakrishnan R, Kane RL Association of frailty with survival: a systematic literature review. Ageing Res Rev 2013; 12 (2): 719-36.

25. Garrido M, Serrano MD, Bartolomé R, Martínez-Vizcaíno V. Diferencias en la expresión del síndrome de fragilidad en varones y mujeres mayores institucionalizados sin deterioro cognitivo grave. Rev Esp Geriatr Gerontol 2012; 47 (6): 247-53.

26. Sousa-Santos AR, Afonso C, Moreira P, Padrão P, Santos A, Borges $\mathrm{N}$ et al. Weakness: The most frequent criterion among pre-frail and frail older Portuguese.
Arch Gerontol Geriatr 2018; 74: 162-68.

27. Gozález-Mechán MC, Leguía-Cerna J, Díaz-Vélez C. Prevalence and factors associated with fragility syndrome in older adults attending the EsSalud primary health care service, January - April 2015. Chiclayo, Peru. Horiz. Med 2017; 17 (3): 35-42

28. Op het Veld LPM, van Rossum E, Kempen GIJM, de Vet HCW, Hajema KJ, Beurskens AJHM. Fried phenotype of frailty: cross-sectional comparison of three frailty stages on various health domains. BMC Geriatr 2015; 15: 77. 